\title{
Analysis of MHD Mixed Convection Flow Within a Square Enclosure Containing a Triangular Obstacle
}

\author{
Kakali Chowdhury ${ }^{1}$, Md. Abdul Alim ${ }^{2}$ \\ ${ }^{1}$ Electrical and Computer Engineering Department, Presidency University, Dhaka, Bangladesh \\ ${ }^{2}$ Mathematics Department, Bangladesh University of Engineering and Technology, Dhaka, Bangladesh
}

Email address:

kakalishome@yahoo.com (K. Chowdhury), maalim@math.buet.ac.bd (Md. A. Alim)

To cite this article:

Kakali Chowdhury, Md. Abdul Alim. Analysis of MHD Mixed Convection Flow Within a Square Enclosure Containing a Triangular Obstacle. American Journal of Applied Mathematics. Vol. 3, No. 6, 2015, pp. 288-296. doi: 10.11648/j.ajam.20150306.18

\begin{abstract}
Finite element method is used to solve two-dimensional governing mass, momentum and energy equations for steady state, mixed convection problem inside a lid driven square enclosure containing a triangular hot obstacle located at the centre of the enclosure. The enclosure top wall is adiabatic while the bottom wall and triangular obstacle are maintained at a uniform temperature higher than the vertical side walls. The left vertical wall is moving with a uniform velocity by unity from bottom to top. All solid boundaries are in no slip condition. The aim of the study is to describe the effect of magnetic field, Prandtl number and the size of triangular obstacle on mixed convection fluid flow, heat transfer and temperature of the fluid. The investigation is conducted for various values of magnetic parameter $H a$, obstacle size (area) $A$, Richardson number $R i$, and Prandtl number Pr. Various results such as streamlines, isotherms, heat transfer rates in terms of average Nusselt number $N u$, and average temperature $\theta_{a v}$ of the fluid in the enclosure are presented for different parameters. It is observed that the obstacle size and dimensionless parameters $\mathrm{Ha}$ and $\mathrm{Pr}$ have significant effect on both the flow and thermal fields. The results also indicate that the average Nusselt number at the heated surface is strongly dependent on the configurations of the system studied under different geometrical and physical configurations.
\end{abstract}

Keywords: Magnetohydrodynamic, Mixed Convection, Square Enclosure, Triangular Obstacle

\section{Introduction}

The study of mixed convection of electrically-conducting fluid in the magnetohydrodynamic (MHD) devices such as coolers of nuclear reactors, thermal insulators and microelectronic devices should account for the effect of a transverse magnetic field on the fluid flow and the heat transfer mechanism. It is found that the fluid flow experiences a Lorentz force due to the influence of magnetic field.

Combined free and forced convective flow in lid driven cavities occurs as a result of two competing mechanisms. The first one is due to shear flow caused by the movement of one of the walls of the enclosure, while the following one is due to buoyancy flow produced by thermal non-homogeneity of the enclosure boundaries. Analysis of mixed convective flow in a lid-driven enclosure finds applications in material processing, flow and heat transfer in solar ponds, dynamics of lakes, reservoirs and cooling ponds, crystal growing, float glass production, metal casting, food processing, galvanizing, and metal coating.
There are many investigations on mixed convective flow in lid-driven cavities. Many different configurations and combinations of thermal boundary conditions have been considered and analyzed by various investigators.

\section{Literature Review}

Alchaar et al [1] studied natural convection heat transfer in a rectangular enclosure with a transverse magnetic field. In this paper the effect of, transverse magnetic field on buoyancydriven convection in a shallow rectangular cavity is numerically investigated. They found that at high Hartmann number, the velocity gradient in the core revealed by both analytical and numericalanalysesisconstant outside the two Hartmann layers at the vicinity of the walls normal tothe magnetic field. Akhter and Alim [2] studied the effects of pressure work on natural convection flow around a sphere with radiation heat loss. They found that increasing Prandtl number leads to decrease the velocity and temperature, increase the skin friction and reduce the rate of heat transfer. Mousa [3] 
investigated the modeling of laminar buoyancy convection in a square cavity containing an obstacle. He found that in case of low Rayleigh numbers $\left(10^{2}-10^{4}\right)$, the rate of heat transfer decreases when the aspect ratio of the adiabatic square obstacle increases. Nasrin[4] investigated mixed magnetoconvection in a lid driven cavity with a sinusoidal wavy wall and a central heat conducting body. She considered a heat conducting square body located at the center of cavity. The cavity horizontal walls are perfectly insulated while the corrugated right vertical surface is maintained at a uniform temperature higher than the left lid. She found that the influence of $\mathrm{Ha}$ does not affect significantly the thermal current activities. But the flow pattern changes dramatically owing to the hindrance of the imposed magnetic field. Aydin [5] studied the effects of moving wall on

aiding and opposing mechanisms of mixed convection in a shear and buoyancy driven cavity. Oztop and Dagtekin [6] extended this idea to two-sided lid driven cavity. They investigated on mixed convection in two-sided lid-driven differentially heated square cavity. Saha et al [7] investigated numerically mixed convection heat transfer in a lid driven cavity with wavy bottom surface. They found that Reynolds number, Grashof number and the number of undulations of the wavy surface have significant effect on the flow fields, temperature distributions and heat transfer in the cavity. Oztop et al [8] studied numerically MHD mixed convection in a liddriven cavity with corner heater. They reported that magnetic field plays an important role to control heat transfer and fluid flow. They showed that heat transfer decreases with increasing of Hartmann number and the rate of reduction is higher for high values of the Grashof number. Rahman and Alim [9] investigated MHD mixed convection flow in a vertical liddriven square enclosure including a heat conducting horizontal circular cylinder with Joule heating. They reported that mixed convection parameter $R i$ affects significantly on the flow structure and heat transfer inside the enclosure and the overall heat transfer decreases with the increase of joule heating parameter $J$. Moallemi and Jang [10] studied numerically mixed convective flow in a bottom heated square lid-driven enclosure. They investigated the effect of Prandtl number on the flow and heat transfer process. Prasad and Koseff [11] reported experimental results for mixed convection in deep liddriven cavities heated from below. They observed that the heat transfer is rather insensitive to the Richardson number. YadollahiFarsani and Ghasemi [12] investigated magnetohydrodynamic mixed convective flow in a cavity. They used a cavity of which lower surface is heated from below whereas other walls of the cavity are thermally isolated. They found that as Hartmann number increases the Nusselt number, representing heat transfer from the cavity decreases. In the present paper the main objective is to examine the fluid flow and heat transfer in a lid-driven square enclosure containing a triangular obstacle in presence of magnetic field.

\section{Governing Equations}

In the present problem, the flow is considered to be steady, two-dimensional, laminar, and incompressible and there is no viscous dissipation. The gravity force acts in the vertically downward direction, fluid properties are constant and fluid density variations are neglected except in the buoyancy term. Radiation effect is neglected. Under the usual Boussinesq approximation, the governing equations for the present problem can be described in dimensionless form by the following equations.

$$
\begin{aligned}
& \frac{\partial \boldsymbol{U}}{\partial \boldsymbol{X}}+\frac{\partial \boldsymbol{V}}{\partial \boldsymbol{Y}}=0 \\
& \boldsymbol{U} \frac{\partial \boldsymbol{U}}{\partial \boldsymbol{X}}+\boldsymbol{V} \frac{\partial \boldsymbol{U}}{\partial \boldsymbol{Y}}=-\frac{\partial \boldsymbol{P}}{\partial \boldsymbol{X}}+\frac{1}{\operatorname{Re}}\left(\frac{\partial^{2} \boldsymbol{U}}{\partial \boldsymbol{X}^{2}}+\frac{\partial^{2} \boldsymbol{U}}{\partial \boldsymbol{Y}^{2}}\right) \\
& \boldsymbol{U} \frac{\partial \boldsymbol{V}}{\partial \boldsymbol{X}}+\boldsymbol{V} \frac{\partial \boldsymbol{V}}{\partial \boldsymbol{Y}}=-\frac{\partial \boldsymbol{P}}{\partial \boldsymbol{Y}}+\frac{1}{\operatorname{Re}}\left(\frac{\partial^{2} \boldsymbol{V}}{\partial \boldsymbol{X}^{2}}+\frac{\partial^{2} \boldsymbol{V}}{\partial \boldsymbol{Y}^{2}}\right)+\boldsymbol{R} \boldsymbol{i} \boldsymbol{\theta}-\frac{\boldsymbol{H} \boldsymbol{a}^{2}}{\operatorname{Re}} \boldsymbol{V} \\
& \boldsymbol{U} \frac{\partial \boldsymbol{\theta}}{\partial \boldsymbol{X}}+\boldsymbol{V} \frac{\partial \boldsymbol{\theta}}{\partial \boldsymbol{Y}}=\frac{1}{\operatorname{RePr}}\left(\frac{\partial^{2} \boldsymbol{\theta}}{\partial \boldsymbol{X}^{2}}+\frac{\partial^{2} \boldsymbol{\theta}}{\partial \boldsymbol{Y}^{2}}\right)
\end{aligned}
$$

The dimensionless variables are defined as:

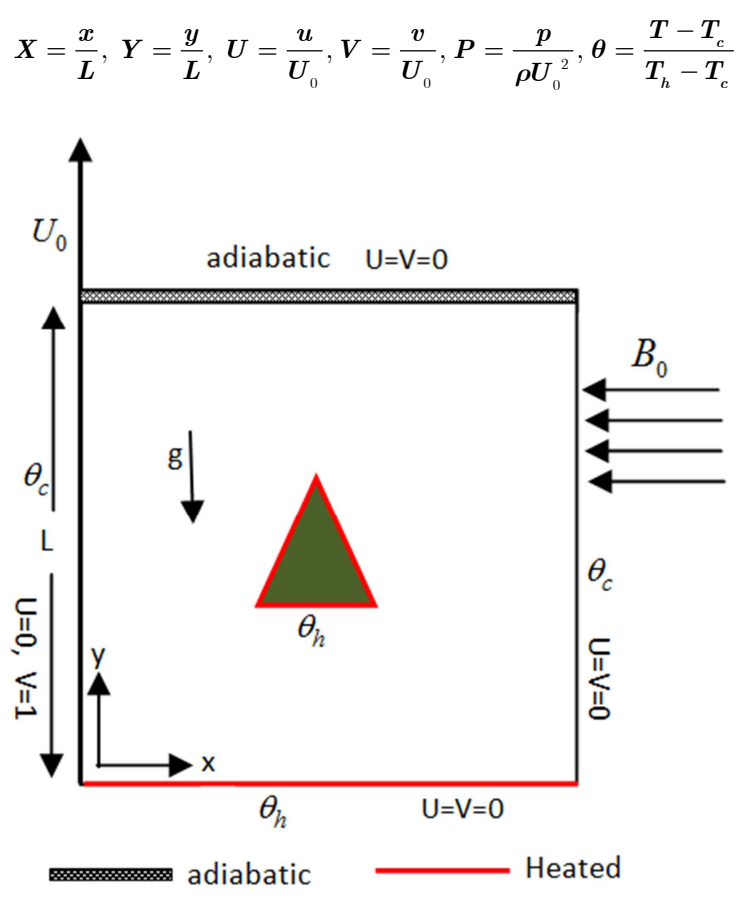

Fig. 1. Schematic diagram of the physical system.

The governing parameters in the preceding equations are the Reynolds number Re, Grashof number $G r$, Hartmann number $\mathrm{Ha}$, Prandtl number Pr, Richardson number $\mathrm{Ri}$ which are defined in the following:

$$
\operatorname{Re}=\frac{U_{0} L}{v}, G r=\frac{g \beta \Delta T L^{3}}{v^{2}}, H a^{2}=\frac{\sigma B_{0}{ }^{2} L^{2}}{\mu}, \operatorname{Pr}=\frac{v}{\alpha}, R i=\frac{G r}{\operatorname{Re}^{2}}
$$

The associated dimensionless boundary conditions are $U=0, V=1, \theta=0$ at the left vertical wall

$U=0, V=0, \theta=0$ at the right vertical wall

$U=0, V=0, \theta=1$ at the bottom wall and triangular obstacle 
surface

$U=0, V=0, \frac{\partial \theta}{\partial Y}=0$ at the top wall. The average Nusselt number at the heated wall of the enclosure is defined as $N u=-\frac{1}{L_{h}} \int_{0}^{L_{h} / L} \frac{\partial \theta}{\partial X} d Y$ and the bulk average temperature in the enclosure is defined as $\theta_{a v}=\int_{0}^{S} \frac{\theta}{S} d S$

\subsection{Geometry of the Problem}

The physical model considered here is shown in fig. 1 along with the important geometric parameters. A Cartesian coordinate system is used with the origin at the lower left corner of the computational domain. It consists of a vertical lid driven square enclosure with sides of length unity, filled with an electrically conducting fluid and containing a triangular obstacle of area $A$. A uniform magnetic field $B_{0}$ is applied horizontally in the direction opposite to X-axis. Top wall is assumed to be adiabatic, both the left and right walls are maintained at a constant temperature $\theta_{c}$, the bottom wall and the triangular obstacle are maintained at a constant temperature $\theta_{h}$ such that $\theta_{h}>\theta_{c}$. The left wall of the enclosure is allowed to move in its own plane with a constant velocity of unity from bottom to top. The Prandtl number Pr of the working fluid varies from 0.03 to 5.43. All other physical properties of the fluid are assumed to be constant except density variation in the body force term of the momentum equation according to the Boussinesq approximation.

\subsection{Numerical Validation}

To validate the present numerical code, the results for mixed convection flow in an enclosed enclosure with heated upper wall have been compared with those obtained by Saha et al [7]. The comparison of the results obtained from present code with those of Saha et al is demonstrated for two different Grashof numbers $G r=10^{2}$ and $10^{6}$ at $\mathrm{Re}=10^{3}$ in fig.2. As seen from this figure, the obtained results show very good agreement.

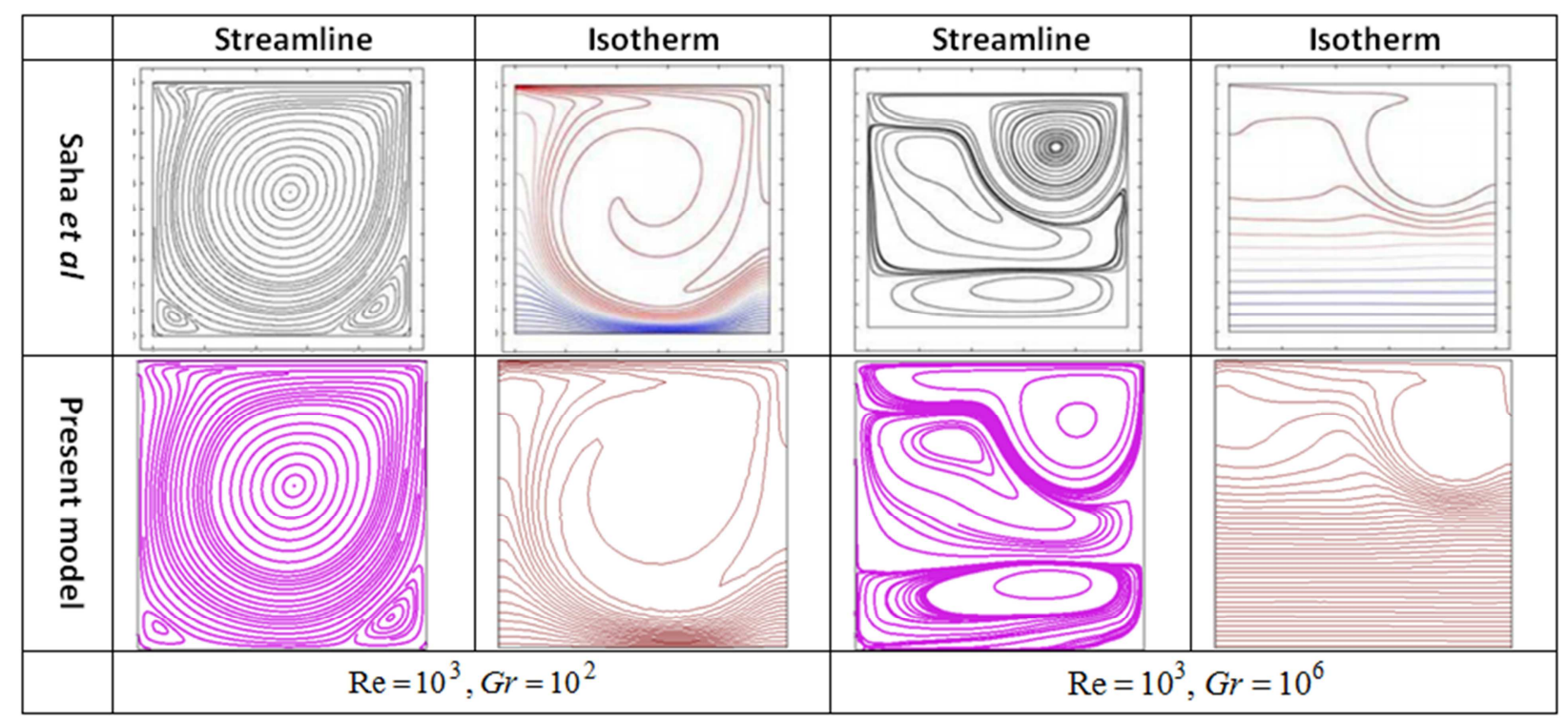

Fig. 2. Comparison of streamlines and isotherms between present model (lower) and Saha et al (upper) at $\mathrm{Re}=10^{3}$ while Gr $=10^{2}$ and $10^{6}$.

\subsection{Numerical Technique}

The numerical procedure used in this work is based on the Galerkin weighted residual method of finite element formulation. The application of this technique is well described by Taylor and Hood [13] and Dechaumphai [14]. In this method, the solution domain is discretized into finite element meshes, which are composed of non-uniform triangular elements. Then the nonlinear governing partial differential equations (i.e. mass, momentum and energy equations) are transferred into a system of integral equations by applying Galerkin Residual method. The integration involved in each term of these equations is performed by using Gauss's quadrature method. The nonlinear algebraic equations so obtained are modified by imposition of boundary conditions. These modified nonlinear equations are transferred into linear algebraic equations by NewtonRaphson iteration. Finally, these linear equations are solved by using Triangular Factorization method.

\section{Results and Discussion}

A numerical study has been performed through finite element method to analyze the laminar mixed convection heat transfer and fluid flow in a lid driven square enclosure containing a triangular obstacle. Effects of the dimensionless parameters such as Richardson number $R i$, Prandtl number $\operatorname{Pr}$ and area $A$ of triangular obstacle on heat transfer and fluid flow of the enclosure have been analyzed. The results are presented in two parts. The first part has focused on flow and temperature fields which explain the streamlines and isotherms for different cases. The other part has focused on 
heat transfer and temperature through the explanation of average Nusselt number at the hot wall and average fluid temperature in the enclosure. The range of Richardson number $R i$ for this investigation is chosen from 0.1 to 10 to obtain the results of forced, mixed and natural convection dominated region based on $R i=\frac{G r}{R^{2}}$. The range of Prandtl number $\operatorname{Pr}$ is chosen from 0.03 to 5.43 to obtain the results for various liquids. Such as 0.03 for liquid metals, 0.71 for air, 2.56 for water at $70^{\circ} \mathrm{C}$ and 5.43 for water at $30^{\circ} \mathrm{C}$.

\subsection{Effect of Hartmann Number}

For the variations of $H a$ and $R i$, the overall features of the streamlines are predicted in fig.3 where $\operatorname{Re}=100, \operatorname{Pr}=0.71, A=0.08$ are kept fixed. In absence of magnetic field and in the forced convection dominated region $(R i=0.1)$, the fluid flow is characterized by a primary rotating uni-cellular vortex occupying the bulk of the enclosure generated by the movement of the left lid. The size of the vortex remains unaffected, but the core of the vortex shifted towards the left top corner of the enclosure with increasing $\mathrm{Ha}$ up to 40 and the core is divided into two parts positioned at the bottom and top near to left wall for higher value of $\mathrm{Ha}$. When forced and natural convection become equally dominant, namely, $R i=1$, the characteristics of the velocity field are more similar to the characteristics as $R i=0.1$. In addition, a minor vortex is formed at $H a=0$ and $H a=20$. When $R i=10$, i.e. the effect of forced convection is very much less compared to natural convection effect, the velocity field forms two large bi-cellular vortices at $H a=0$. In addition, a minor vortex is formed adjacent to moving wall. Furthermore, the size of the middle vortex decreases and the minor vortex becomes larger as $H a$ increases from 0 to 60 . This is because of the application of a transverse magnetic field which tends to slow down the movement of the buoyancy-induced flow in the enclosure.The corresponding effects on the temperature field are shown in fig 4. The isotherms are almost parallel to the both vertical walls for higher value of $\mathrm{Ha}$ at all $\mathrm{Ri}$, indicating that most of the heat transfer process is carried out by conduction. However, some deviations in the conduction dominated isothermal lines are initiated near the left top surface of the enclosure for $H a=40$. This distortion increases quickly with decreasing $H a$ at $R i=0.1$. The isothermal lines dominated by conduction and mixed convection heat transfer are observed in the enclosure for different $H a$ at $R i=1$. But when $R i=10$, the temperature field is drastically changed with diminishing $\mathrm{Ha}$. Moreover, the formation of the thermal boundary layers near the two vertical cold walls is to be initiated for the lower value of $H a$. This is owing to the dominating influence of the convective current in the enclosure. In order to evaluate how the presence of the magnetic field effects the heat transfer rate along the hot wall, average Nusselt number is plotted as a function of Richardson number $R i$ and Hartmann number $\mathrm{Ha}$ as shown in fig 5(a). It is observed that the average $\mathrm{Nu}$ decreases in the forced to mixed convection dominated region for all values of $\mathrm{Ha}$. But from mixed to natural convection dominated region it increases gradually in absence of magnetic field and for low values of Ha. As Ha increases, the increasing rate of $\mathrm{Nu}$ becomes lower and at $\mathrm{Ha}=60$ it is nominal. Moreover, $N u$ is always higher for small values of $\mathrm{Ha}$. Fig.5 (b) exposes the influence of $\mathrm{Ha}$ on average temperature $\theta_{a v}$ of the fluid in the enclosure along with $R i$. For lower Hartmann number $(H a=0, H a=20), \theta_{a v}$ increases very rapidly in all the regions but for higher Ha, $\theta_{a v}$ increases almost linearly.

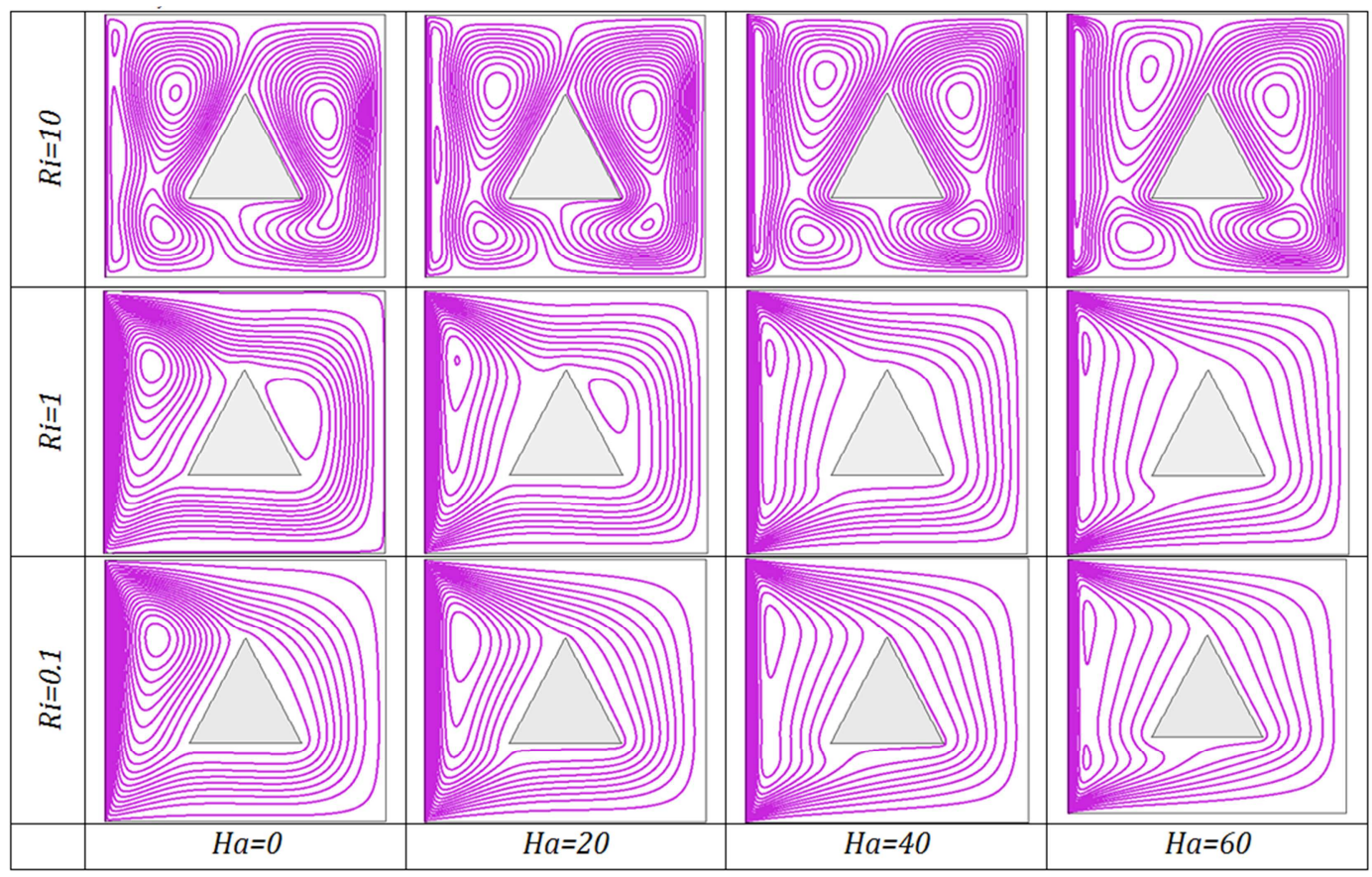

Fig. 3. Streamlines for different values of $H a$ and $R i$, while $\operatorname{Re}=100, \operatorname{Pr}=0.71, A=0.08$. 


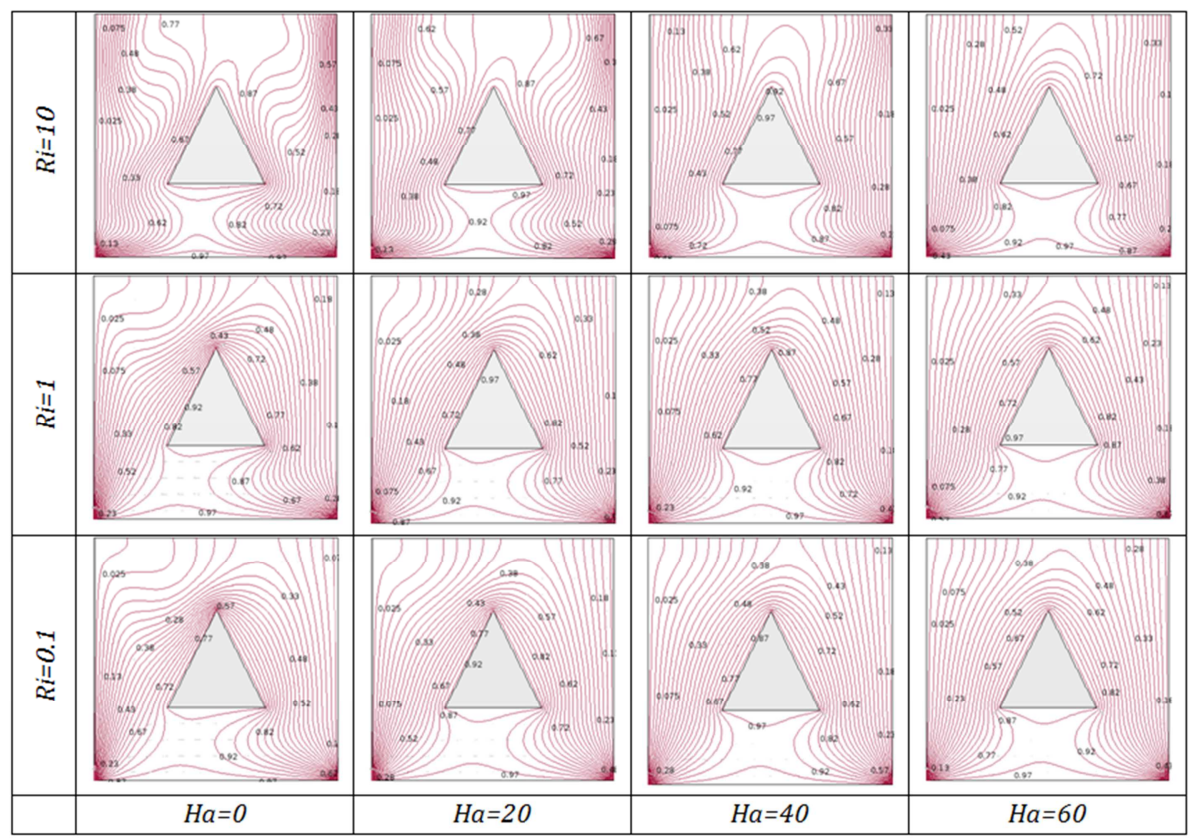

Fig. 4. Isotherms for different values of $H a$ and $R i$, while $\operatorname{Re}=100, \operatorname{Pr}=0.71, A=0.08$.

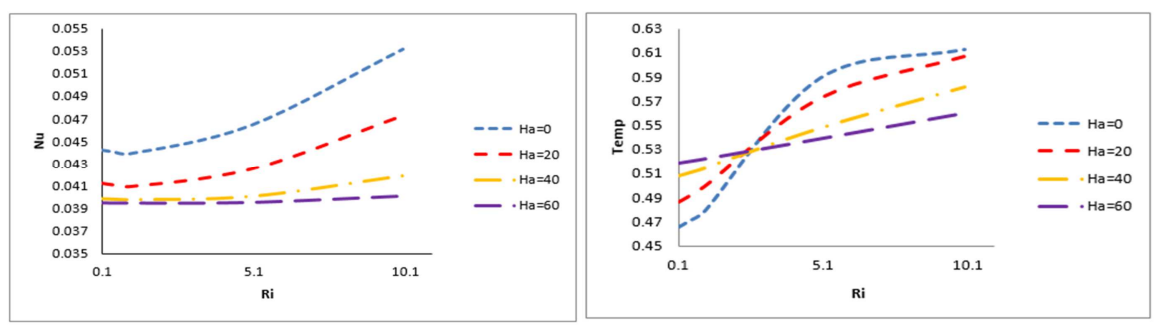

Fig. 5. Effect of Hartmann number on (a) average Nusselt number (b) average temperature while $\operatorname{Re}=100, \operatorname{Pr}=0.71, \quad A=0.08$.

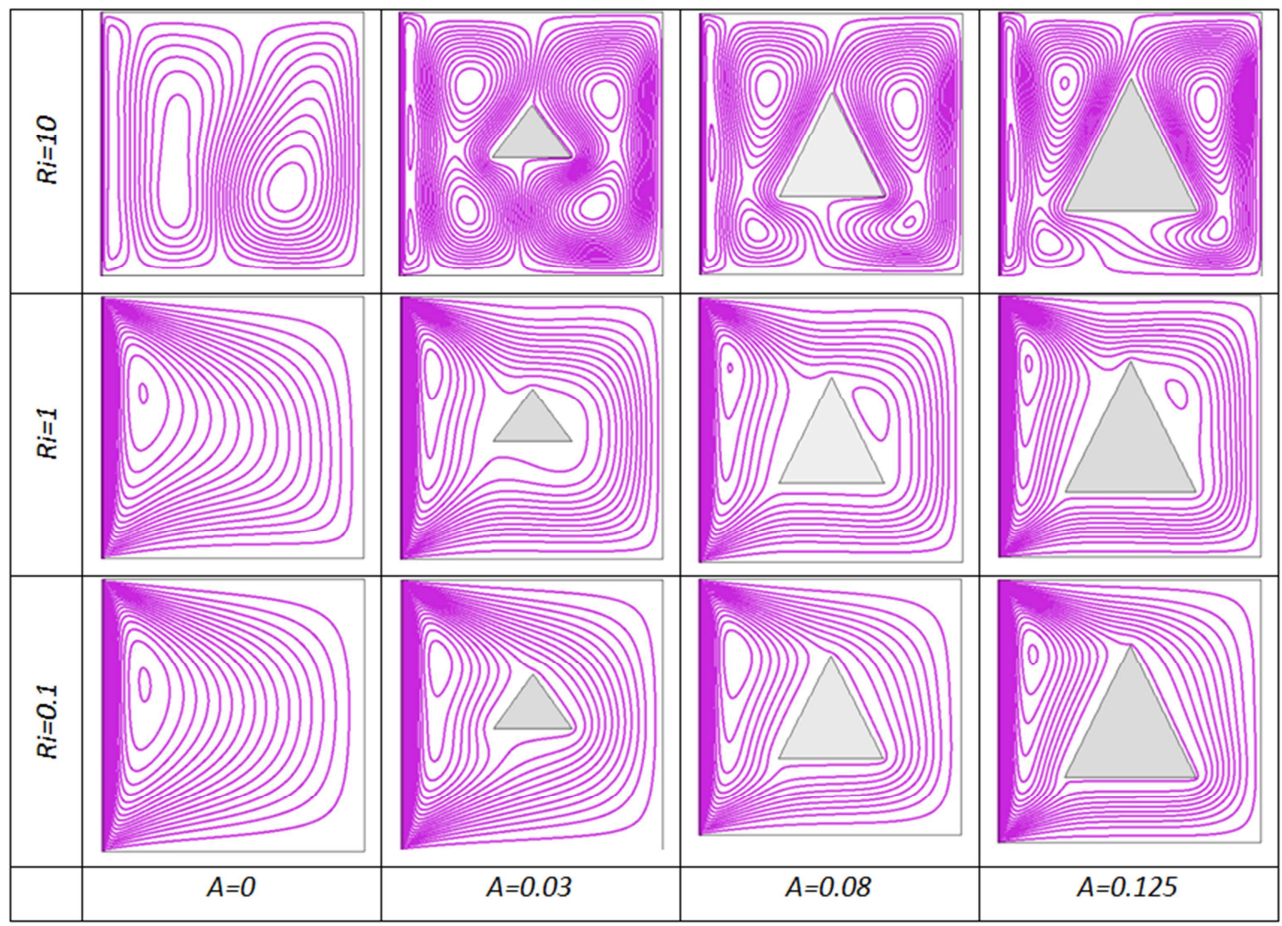

Fig. 6. Streamlines for different values of obstacle area and Richardson numbers, while $\operatorname{Re}=100, \operatorname{Pr}=0.71, H a=20$. 


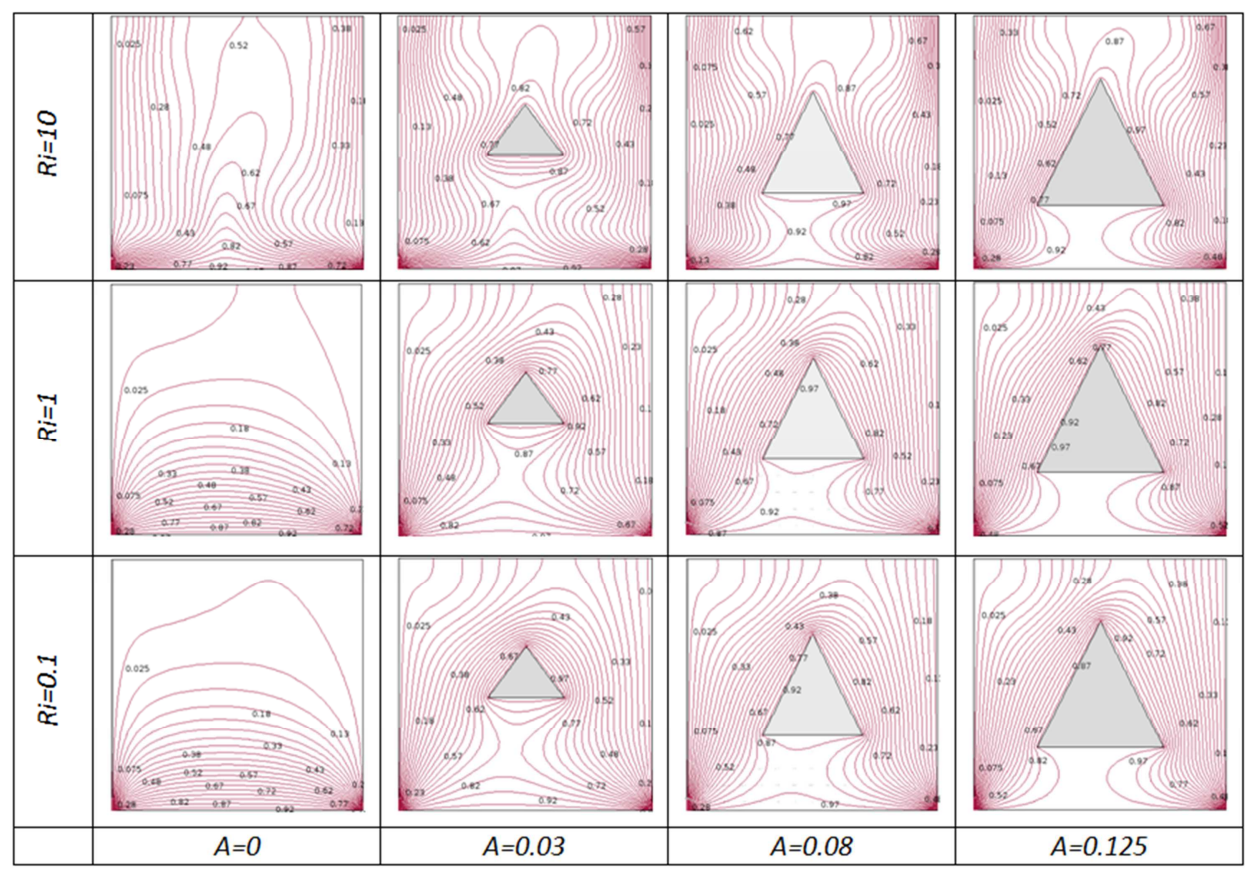

Fig. 7. Isotherms for different values of obstacle area and Richardson numbers, while $\operatorname{Re}=100, \operatorname{Pr}=0.71, H a=20$.

\subsection{Effect of Triangular Obstacle Size}

Effect of obstacle size on streamlines and isotherms is presented in fig. 6 and fig. 7 for $\mathrm{Re}=100, \mathrm{Pr}=0.71, \mathrm{Ha}=20$ and various $R i \quad(R i=0.1,1,10)$. The flow structure for the four different values of triangular obstacle area in the forced convection dominated region has been shown in the bottom row of fig.6. In absence of obstacle, it is seen that a uni-cellular vortex appears nearer to the left vertical moving wall, which is owing to the effect of shear force and buoyancy force.
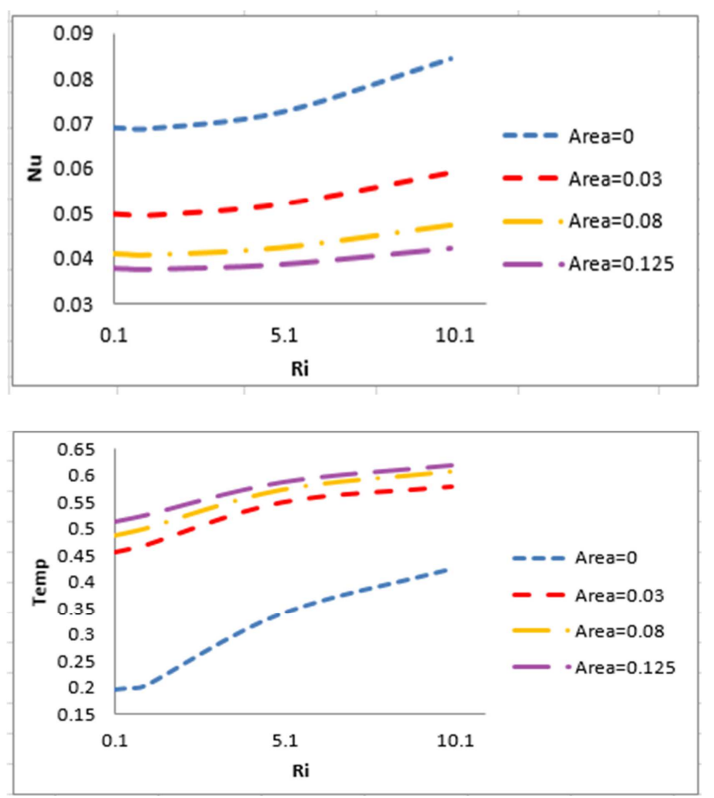

Fig. 8. Effect of hot triangular obstacle area on (a) average Nusselt number; (b) average temperature while $\mathrm{Re}=100, \mathrm{Pr}=0.71, H a=20$.

For $A=0.03$, the obstacle divides the vortex occupying the space in the centre of the enclosure. As area increases, the density of streamlines near the left moving wall increases and the counterpart of right wall decreases. This is due to the increasing size of the triangular obstacle decreases the space available for the vortices. For $R i=1$, it is seen from fig. 6 that the forced convection effect is present, but remains relatively weak at higher values of $A$ since the uni-cellular vortex characterizing the imposed flow are still dominant. For higher values of A, a tiny vortex is seen at the top right corner of the enclosure. Further increase of $R i$ to 10 , gives three vertical vortices for $A=0$. Among these, the left one is minor which is adjacent to the moving wall and the vortex adjacent to the right wall is the larger. As area increases all the vortices become denser and each of the larger two vortices gradually becomes bi-cellular vortices. The isotherms in absence of triangular obstacle and for the three values of $R i$ are shown in the left column of fig.7. At $R i=0.1$ and $A=0$, the high temperature region is concentrated near the hot wall and isothermal lines have taken shape from linear to semi circular to the heated surface of the enclosure indicating forced convection heat transfer. As $R i$ increases, the nonlinearity of the isotherms becomes higher and plume formation is profound in the centre and other isotherms tend to be parallel to the vertical wall indicating the well established natural convection. Plots of the average Nusselt number $N u$ at the heated wall and average temperature $\theta_{a v}$ of the fluid in the enclosure as a function of $R i$ and $A$ have been shown in Fig 8. Nu at the heated wall decreases very slightly in the forced to mixed convection dominated region but from mixed to natural convection dominated region it increases monotonically for all values of $A$, this is due to increasing $R i$ enhances convective heat transfer. Moreover, $N u$ is always higher for small values of $A$. It is seen that the average temperature of the fluid in the enclosure increases 
with increasing value of $R i$ for all values of $A$. At $A=0$ the average temperature of the fluid in the enclosure is reasonably lower than other values of $A$. In presence of obstacle, fluid temperature increases abruptly and with the increasing value of $A$, fluid temperature increases gradually.
This shows that presence and increasing size of triangular obstacle has great effect on fluid temperature. This can be attributed to the fact that a large centered triangular obstacle narrows the regions available for warm fluid flows and enhances fluid temperature.

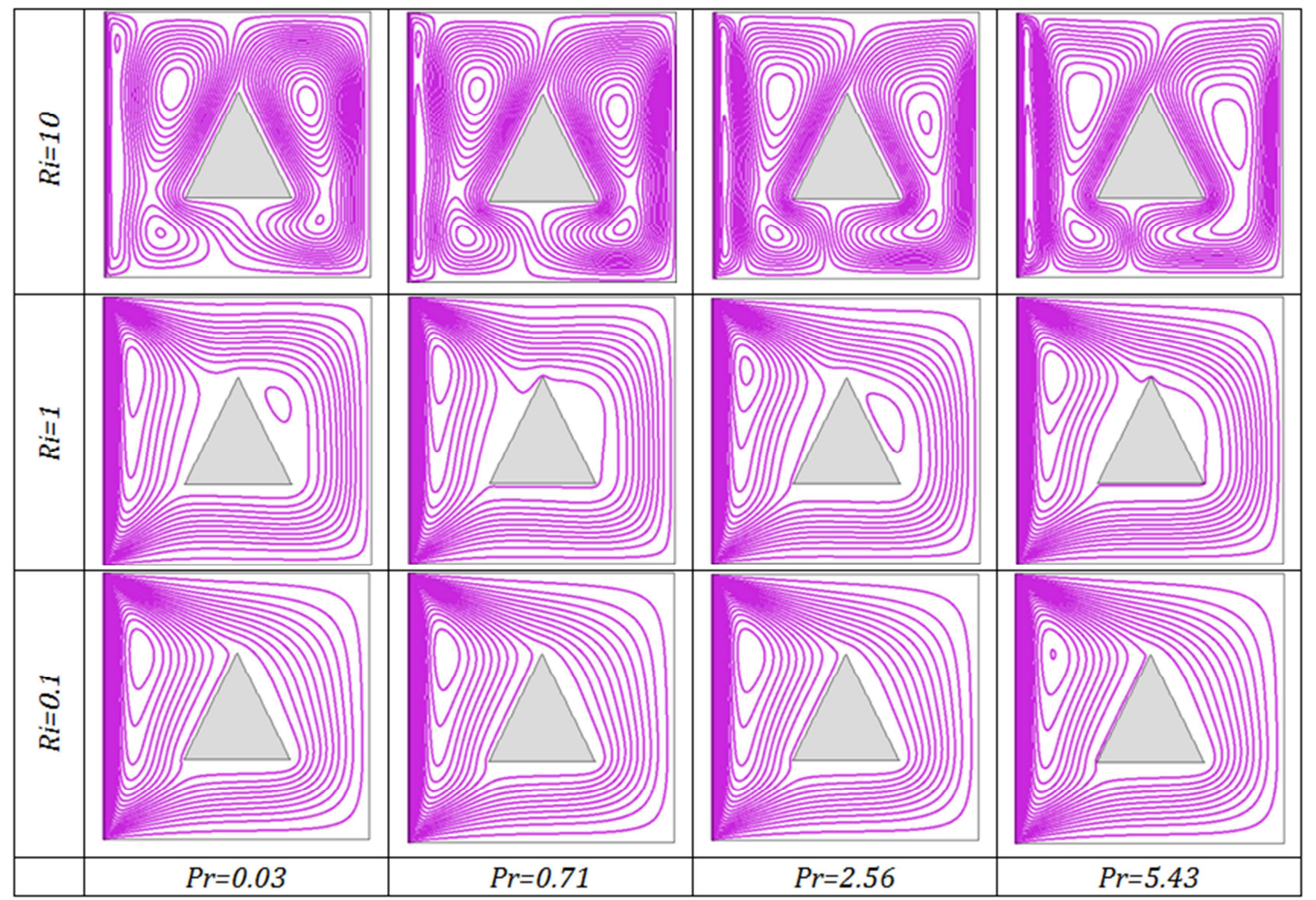

Fig. 9. Streamlines for different Prandtl numbers and Richardson numbers, while $\mathrm{Re}=100, A=0.08, H a=20$.

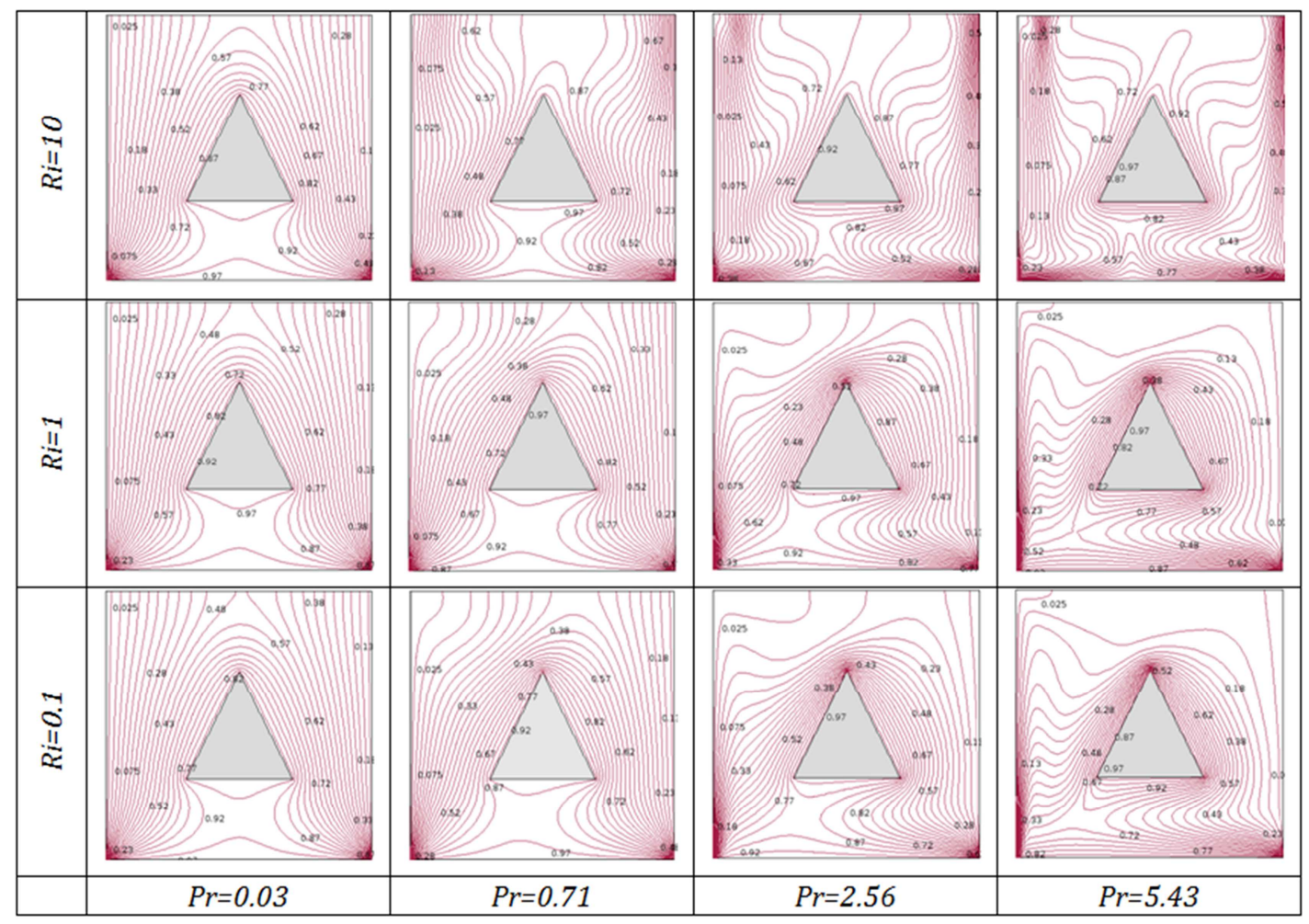

Fig. 10. Isotherms for different Prandtl numbers and Richardson numbers, while $\mathrm{Re}=100, A=0.08, H a=20$. 


\subsection{Effect of Prandtl Number}

The influence of Prandtl number on the flow field at three different values of $R i$ is shown in fig.9 while $\mathrm{Re}=100, A=0.08, H a=20$. The streamlines for various values of $\operatorname{Pr}(\operatorname{Pr}=0.03,0.71,2.56,5.43)$ in the forced convection dominated region is shown in the bottom row of fig.9. Here the fluid flow is due to the shear induced force by the moving lid only. Next at $R i=1$, the balance between the shear and buoyancy effect is manifested in the formation of a new small vortex near the obstacle. As $R i$ rises further to 10 , the heat transfer is mostly by natural convection in the enclosure. Three vertical vortices are formed in two sides of the obstacle. Among these, the larger two are bi-cellular vortices and the smaller one is adjacent to lid driven wall. As Pr increases the smaller vortex becomes larger. Temperature field has been simulated using isotherms for the mentioned parameters.
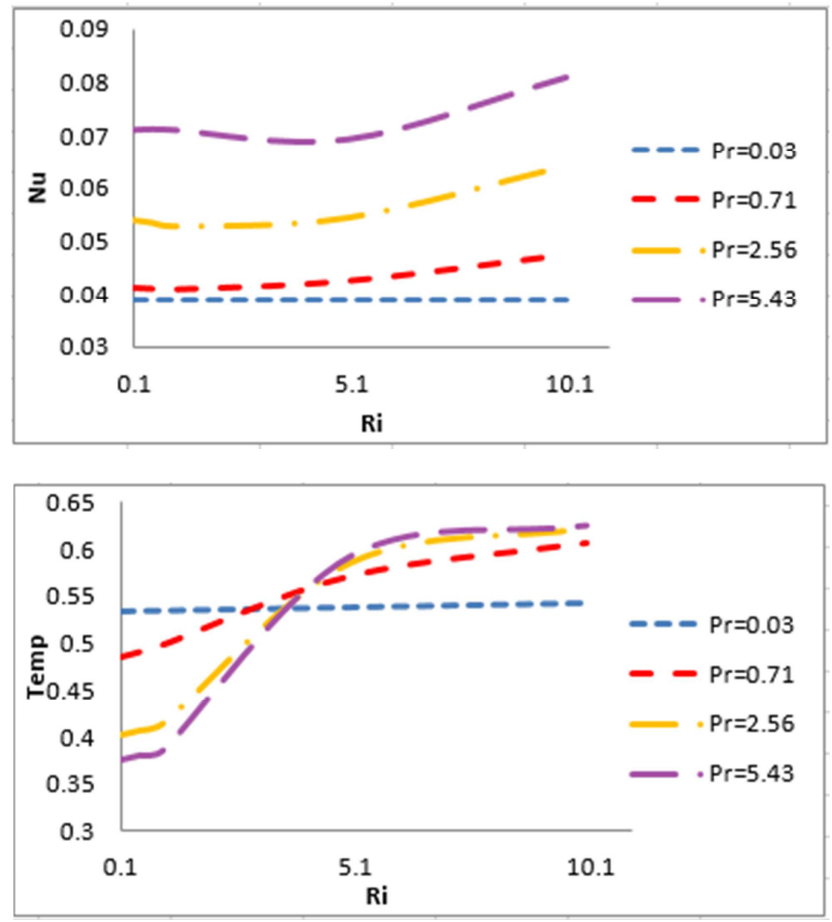

Fig. 11. Effect of Prandtl number on (a) average Nusselt number (b) average temperature while $\mathrm{Re}=100, \mathrm{~A}=0.08, \mathrm{Ha}=20$.

Effect of Prandtl number on isotherms is presented in fig.10 for $\mathrm{Re}=100, \mathrm{Ha}=20, A=0.08$ and various $\operatorname{Pr}(\operatorname{Pr}=0.03,0.71,2.56,5.43)$. For $P r=0.03$ the isotherms nearer to side walls become almost parallel to the vertical walls resembling the conduction like heat transfer in the enclosure and the isotherms adjacent to obstacle are mostly nonlinear. Thus when the fluid is considered as liquid metal it has almost no effect on convection process. As Pr increases (the fluid is considered as air and water at various temperatures) the bending of isothermal lines increases and they are accumulating near the obstacle in the forced convection dominated region. But in the natural convection dominated region the isotherms depart from the hot wall and begin to crowd near the cold walls forming thermal boundary layers in the enclosure. As Pr increases the isotherms above the obstacle form a plume. Plots of the average Nusselt number $N u$ at the heated wall and average temperature $\theta_{a v}$ of the fluid in the enclosure as a function of Riand $\operatorname{Pr}$ have been shown in Fig 11. For $\operatorname{Pr}=0.03$ when the fluid is considered as liquid metal, the average Nusselt number remains almost the same in all the regions from forced to mixed and mixed to natural convection. For $P r=0.71$ and 2.56 , when fluid is considered as air and water at $70^{\circ} \mathrm{C}, N u_{a v}$ decreases in forced to mixed convection dominated region slightly but it increases abruptly in the mixed to natural convection dominated region. For $\operatorname{Pr}=5.43$ when fluid is considered as water at $30^{\circ} \mathrm{C}$, the characteristics of $N u_{a v}$ is oscillatory. On the other hand, average temperature of the fluid $\theta_{a v}$ increases for increasing value of $R i$ but it decreases with the increasing value of $\operatorname{Pr}$. For $\operatorname{Pr}=0.03, \theta_{a v}$ remains almost the same in all the regions. When $\operatorname{Pr}=0.71$ or above, $\theta_{a v}$ increases slightly in the forced to mixed convection dominated region but it increases abruptly in the mixed to natural convection dominated region.

\section{Conclusion}

From this investigation, it is found that all the parameters magnetic field, Prandtl number, Richardson number and size of triangular obstacle play significant role on average heat transfer and fluid temperature. The major conclusions have been drawn as followings:

- Heat transfer rate is higher in absence of magnetic field. Presence of magnetic field decreases $N u$ drastically, but increasing value of Ha decreases $N u$ gradually. $\theta_{a v}$ increases abruptly for lower Ha but it increases linearly for higher Ha.

- The presence of triangular obstacle decreases $N u$ drastically. As area of obstacle increases, $N u$ decreases gradually. $\theta_{a v}$ enhances abruptly in presence of obstacle and as the area of obstacle increases, $\theta_{a v}$ of the fluid increases gradually.

- Increasing value of $\mathrm{Pr}$ increases $\mathrm{Nu}$ and it is higher at $\mathrm{Ri}=10$. For liquid of higher $\operatorname{Pr}, \theta_{a v}$ increases slowly in the region $0.1 \leq R i \leq 1$ but it increases abruptly in the region $1 \leq R i \leq 10$.

\section{Nomenclature}

$c_{p}$ specific heat at constant pressure

g gravitational acceleration $\left[\mathrm{ms}^{-2}\right]$

$h$ convective heat transfer coefficient $\left[\mathrm{Wm}^{-2} \mathrm{~K}^{-1}\right]$

$k$ thermal conductivity of fluid $\left[\mathrm{Wm}^{-1} \mathrm{~K}^{-1}\right]$

$T$ dimensional temperature $[K]$

$\Delta T$ dimensional temperature difference $[K]$ 




\section{Greek Symbols}

$\alpha$ thermal diffusivity $\left[\mathrm{m}^{2} \mathrm{~s}^{-1}\right]$
$\beta$ thermal expansion coefficient $\left[\mathrm{K}^{-1}\right]$
$\rho$ density of the fluid $\left[\mathrm{kgm}^{-3}\right]$
$\mu$ dynamic viscosity of the fluid $\left[\mathrm{m}^{2} \mathrm{~s}^{-1}\right]$
$v$ kinematic viscosity $\left[\mathrm{m}^{2} \mathrm{~s}^{-1}\right]$
$\theta$ non dimensional temperature
$c$ cold
$h$ heated

\section{References}

[1] Alchaar, S., Vasseur, P., Bilgen, E., 1995. Natural convection heat transfer in a rectangular enclosure with a transverse magnetic field," Journal of Heat Transfer, 117 (3), pp. 668 673.

[2] Akhter, T., Alim, M.A., 2010. Effects of pressure work on natural convection flow around a sphere with radiation heat loss. Nonlinear Analysis: Modeling and Control, vol. 15, No. 3, 287-298.

[3] Mousa, M.M. 2010. Modeling of laminar buoyancy convection in a square cavity containing an obstacle. Mathematics Subject Classification: 65M60, 76D05, 80A20.

[4] Nasrin, R., 2011. Mixed magnetoconvection in a lid driven cavity with a sinusoidal wavy wall and a central heat conducting body. Journal of Naval Architecture and Marine Engineering. DOI: 10.3329/ jname.v8il.6793.

[5] Aydin, O. The effects of moving wall on Aiding and opposing mechanisms of mixed convection in a shear and buoyancy driven cavity. Int. Commun. Heat and Mass Transfer 26 (1999) 1019-1028.

[6] Oztop, H.F., Dagtekin, I, 2004. Mixed convection in two-sided lid-driven differentially heated square cavity. Int. J. Heat Mass Transfer 47, 1761-1769.

[7] Saha, L.K., Somadder, M.C., Salah Uddin, K.M. 2013. Mixed convection heat transfer in a lid driven cavity with wavy bottom surface. American Journal of Applied Mathematics 1(5): $92-101$.

[8] Oztop, H.F. Al-Salem, K. Pop, L., 2011. MHD mixed convection in a lid-driven cavity with corner heater. International Journal of Heat and Mass Transfer vol. 54, pp. 3494-3504.

[9] Rahman, M. M., Alim, M. A., 2010. MHD mixed convection flow in a vertical lid-driven square enclosure including a heat conducting horizontal circular cylinder with Joule heating. Nonlinear Analysis: Modeling and Control, Vol. 15, No. 2, 199-211.

[10] Moallemi, M. K., Jang, K. S., 1992. Prandtl number effects on laminar mixed convection heat transfer in a lid-driven cavity. Int. J. Heat Mass Tran., 35, pp. 1881-1892.

[11] Prasad, A.K., Koseff, J.R., 1996. Combined forced and natural convection heat transfer in a deep lid-driven cavity flow. Int $\mathrm{J}$. Heat and Fluid Flow., vol. 17, pp. 460-467.

[12] Yadollahi Farsani, R., Ghasemi, B., 2011. Magnetohydrodynamic mixed convective flow in a cavity. World Academy of Science, Engineering and Technology. Vol $5,11-21$.

[13] Taylor, C, Hood, P. A. numerical solution of the Navier-Stokes equations using finite element technique, Computers \& Fluids 1 (1) (1973) 73-89.

[14] Dechaumphai, P. Finite Element Method in Engineering, $2^{\text {nd }}$ ed. Chulalongkorn University Press, Bangkok, 1999. 